\title{
The neurogenetics and evolution of food-related behaviour
}

\author{
Scott J. Douglas, Ken Dawson-Scully and Marla B. Sokolowski
}

Department of Biology, University of Toronto, 3359 Mississauga Road, L5L 1C6, Canada

\begin{abstract}
All organisms must acquire nutrients from the ambient environment to survive. In animals, the need to eat has driven the evolution of a rich array of complex foodrelated behaviours that ensure appropriate nutrient intake in diverse niches. Here, we review some of the neural and genetic components that contribute to the regulation of food-related behaviour in invertebrates, with emphasis on mechanisms that are conserved throughout various taxa and activities. We focus on synthesizing neurobiological and genetic approaches into a neurogenetic framework that explains foodrelated behaviour as the product of interactions between neural substrates, genes and internal and external environments.
\end{abstract}

\section{Introduction to food-related behaviours}

All life must acquire organic and inorganic substrates as fuel and raw material for energetic, metabolic and physiological processes. Whereas many plants synthesize their constituent organic compounds from carbon dioxide, water and sunlight, animals enact a heterotrophic strategy to gain necessary nutrients. In turn, they have evolved an impressive array of behaviours to detect, capture and ingest food. Broadly, food-related behaviour can be defined as actions elicited in response to hunger or the perception of food, therefore including food-searching activities, intake and post-ingestive behaviours. Multiple internal and external cues influence whether an animal is searching for food or eating at any given time. A major internal parameter is the level of hunger, which is governed by a milieu of regulatory factors interacting within an elaborate homeostatic system [1,2]. External factors include abundance and distribution of food, competitors, risk of predation, season and time of day. In humans, the decision to eat is complicated further by psychological and cultural factors. Owing to the breadth of contributing factors, food-related behaviour represents a rich interface that has been characterized from several different perspectives, including the ecological $[3,4]$, physiological [5], neurobiological [6], psychological [7], endocrinological [8] and genetic [1,2].

A comprehensive description of any behaviour requires insight into the pattern and functioning of the neural circuits that underlie expression of the behaviour. Circuit output, which is often modifiable through experience, depends on parameters such as neuronal membrane

Corresponding author: Sokolowski, M.B. (msokolow@utm.utoronto.ca).

Available online 3 October 2005 properties, neuronal connections and modes of synaptic transmission. Each of these characteristics in turn emerges out of interactions between the environment and genes encoding the myriad of humoral, intercellular and intracellular factors that direct nervous system activity. Examples of molecular players important for representation of behaviour in the nervous system include signalling molecules, receptors, ion channels, intracellular transduction components, and regulators of transcription and protein synthesis.

In the past, the fields of behavioural neurobiology and behavioural genetics have advanced mostly separately, although both have benefited greatly from studies in invertebrates. Neurobiologists have used the small and tractable neural circuits of insects, crustaceans and molluscs, and geneticists have exploited Drosophila melanogaster and Caenorhabditis elegans for their short generation times, high fecundities, high-resolution genetic maps, sequenced genomes and ease of genetic manipulation. From both neural and genetic perspectives, a common theme emerging across invertebrates is the conservation of central regulators of feeding. Nevertheless, owing to the different systems employed in each field, comprehensive models of feeding behaviour have been elusive. For example, although the neuronal dynamics necessary for various ingestion and post-ingestion behaviours have been modelled exquisitely in some invertebrates, little is known of the genetic mechanisms that regulate the development and function of the underlying circuitry. By contrast, genetic analysis of food-related activities in other species has uncovered genes and pathways important for food-search and food-intake behaviours, but a neural context for gene function is lacking owing to the unavailability of circuit information.

In this article, we focus on integration, both mechanistically, in emphasizing the evolutionary conservation of neural and genetic regulators of food-related behaviour across invertebrate species, and practically, in proposing the uniting of neurobiological and genetic paradigms to build neurogenetic (see Glossary) models of feeding behaviour. For the former, invertebrate neural central pattern generators (CPGs) and intracellular cGMPdependent signalling pathways are striking examples of conserved regulators. For the latter, the analysis of gene function in systems traditionally not associated with genetic techniques should help in understanding the relationships between genes, neural substrates and behaviour. As an example of how a neurogenetic synthesis 


\section{Glossary}

Neurogenetics: An approach that examines how genes and molecules contribute to nervous system function and development. Novel genes affecting nervous system operation can be identified by characterizing the genetic basis of heritable phenotypic variation. How a gene influences the nervous system is investigated by determining its expression pattern and the consequences of restricting expression to specific neurons. Combined with electrophysiological and anatomical descriptions, and ablation of specified neurons, integrative models can be developed that depict the emergence of nervous system patterns and dynamics out of specific molecular properties and interactions. Candidate-gene approach: This uses the known function of a gene in one phenotype to hypothesize involvement of that gene in a related phenotype, often in a different organism.

Genomics: The comprehensive study of the sequences, functions and interactions of entire sets of genes and their products.

Transgenic: An organism whose genome has been artificially modified by input of foreign DNA, often of a different species.

Expressed sequence tag (EST): A short string of DNA sequence (typically 200500 base pairs) that is complementary to a portion of a cellular mRNA species. Isolation of ESTs from a tissue reveals which genes are expressed and provides a starting point for compilation of microarrays.

Post-transcriptional interference: Also known as gene silencing, this relies on a battery of techniques that exploit specificity in RNA sequence and protein structure to inhibit either protein synthesis or protein function.

might appear, we describe the regulation of food-related aggregation in C. elegans, where combined analysis of gene and neural function is beginning to set forth an integrative account of this behaviour.

\section{Making connections: examining the neural basis of food-related behaviour}

Elucidation of neural processes underlying natural behaviours has traditionally been achieved under the banner of neuroethology. Studies have often concentrated on small invertebrate circuits, leading to a wealth of knowledge about the neural basis for rhythmic behaviours such as respiration, locomotion and feeding. Systems used to model neural controls of feeding include the stomatogastric ganglion (STG) of the lobsters Panulirus and Homarus [9] and the crab Cancer [10], the buccal ganglia of the gastropods Aplysia and Lymnaea [11], and the subesophageal or frontal ganglia in insects such as the locust Locusta [12] or tobacco horn worm Manduca sexta [13]. These systems have been used to define how properties such as neuronal connections, neurotransmitter identities, membrane characteristics and inter-circuit communication contribute to specific feeding behaviours and to behavioural plasticity. One general finding has been the importance of neuronal pacemakers (i.e. CPGs) to the execution of various rhythmic feeding behaviours. CPGs act in diverse neurobiological contexts such as flight, respiration, locomotion and chewing, and use a conserved set of principles that includes prevalence of synaptic inhibition and an ability to modify output firing patterns depending on the state of the organism [10]. The manner in which CPG organization is transduced into invertebrate feeding activities has been meticulously exposited for several behavioural models, including rhythmic movement of the toothed radula during feeding in the sea slug Aplysia [14] (Figure 1).

Other studies have focused on cellular mechanisms underlying plasticity in CPG-controlled food-related behaviours. Jones and colleagues [15] showed using the pond snail Lymnaea that associative learning correlates

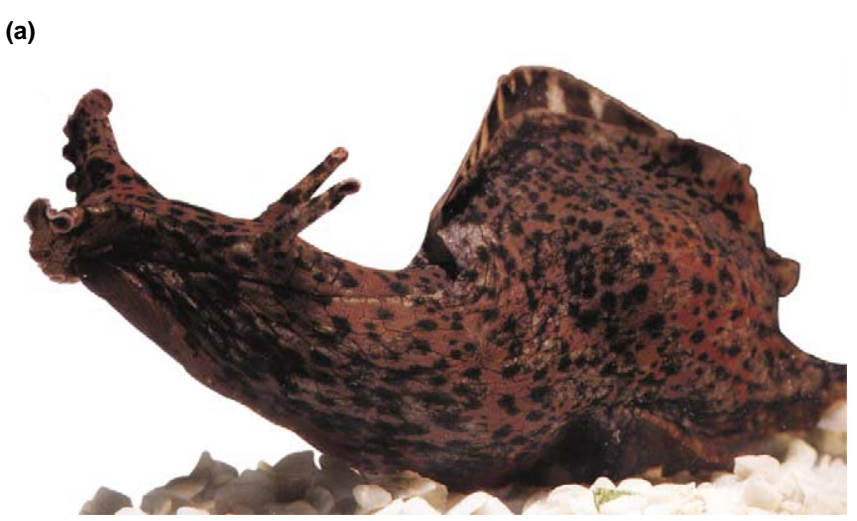

(b)

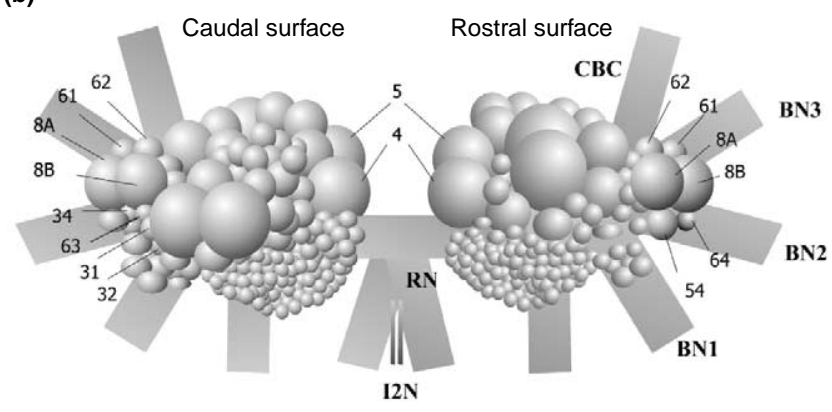

(c)

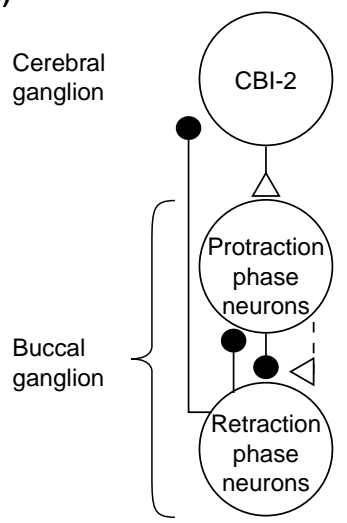

(d)

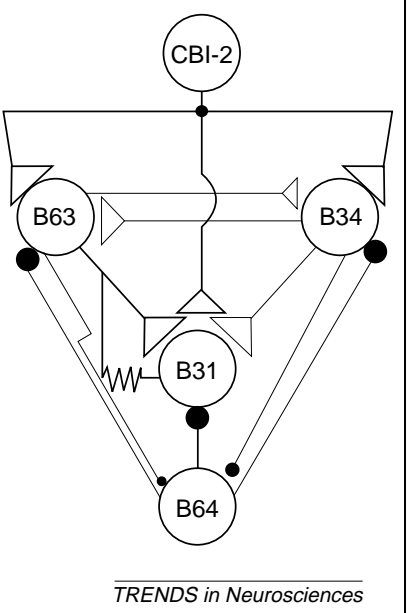

Figure 1. Morphological and neurological coupling of the central pattern generator (CPG) responsible for protraction and retraction movements of the toothed radula during feeding in Aplysia. Food touching the lips or stimulating the interior of the mouth of Aplysia initiates food intake, whereas maintained stimulation triggers repeated rhythmic protraction and retraction movements of the toothed radula. This rhythmic pattern is initiated by a command-like neuron, the cerebral-buccal interneuron 2 (CBI-2). (a) The marine mollusc Aplysia. (b) A morphological map of the rostral and caudal surfaces of the buccal ganglia, indicating the positions of neurons of the CPG involved in protraction and retraction of the radula during ingestion and egestion. Neurons of interest are numbered; nerves innervating the ganglia contain the letter $\mathrm{N}$ in their nomenclature. (c) Relationships among the command-like neuron $\mathrm{CBI}-2$ of the cerebral ganglion and the protraction-phase and retraction-phase neurons in the buccal ganglion. Triangles represent excitatory coupling, dark circles represent inhibitory coupling and the broken line represents a polysynaptic connection. (d) A detailed circuit diagram of the neurons involved in the relationships between protraction and retraction phases, where $\mathrm{CBI}-2$ stimulates the protraction CPG elements B63, B34 and B31 (and also B32, not shown in this figure). The retraction-phase is strongly governed by the CPG element B64, which inhibits all of the protraction-phase interneurons and is itself weakly inhibited by the protraction CPG elements. Triangles, dark circles and lines have the same meanings as in (c), with size corresponding to the strength of the specific connection; the 'resistor' (jagged line) represents electrical coupling. Panel (a) kindly provided by John H. Byrne; (b-d) modified, with permission, from [14]. 
with long-term depolarization of a key modulatory neuron in the circuits regulating ingestive movements. At the same time, phosphorylation of cAMP-response-element binding protein, an important modification for creation of long-term memory, is increased [16]. Another study showed in Aplysia that biting frequency can be enhanced through operant reward learning [17], a method that rewards an animal (in this case with an artificially invoked neural stimulus) for performing an initially neutral action. Once again, changes in the threshold required to produce action potentials in a key regulator of pacemaker activity correlated with behavioural plasticity. Therefore, both the internal dynamics of CPG networks related to food-related behaviours (Figure 1) and the integration of environmental inputs into such circuits have been modelled in invertebrates to provide an understanding of neural bases of feeding at the CPG level.

As mentioned, relatively little is known of the genetic mechanisms that underlie CPG function and plasticity in these invertebrate feeding systems. Nonetheless, the welldefined structure of the neural apparatus associated with feeding behaviours represents an ideal substrate for genetic analysis. Genetic characterization of these systems can identify key regulators of rhythmic behaviours, and the precise roles of these molecules in guiding the output of CPGs. An important question is how CPGs controlling food-related movements of an animal are customized to respond to nutrients in the internal or external environment. That is, do specialized circuit properties responsive to nutrient-related cues modulate a basic, conserved CPG mechanism to direct behaviour towards food-related activities? If so, which genes regulate basic CPG activity and how has the system been modified specifically to tie the neural substrates to food-related behaviour? Next, we present examples of how candidategene approaches and genomic techniques (see Glossary) might be used to dissect the genetics of feeding-related circuitries and address these issues. We then go on to provide an example of how genetic and neurobiological infrastructures can begin to be merged into a multi-tiered, integrative account of food-related behaviour.

\section{Foraging ahead: discovering genes that regulate food-related behaviour}

A central principle of classical genetic analysis is that the identification of genes regulating a biological trait requires heritable variation in the appearance or state of that trait. This phenotypic variation can occur naturally or result from experimentally induced mutations. For food-related behaviour, both natural gene variants and mutations that affect food-search patterns, types of foods preferred and amount eaten per meal have been identified [18-21]. As outlined below, analysing the molecular correlates of phenotypic variation is a powerful means of identifying novel genes and neural substrates important for expression of a behaviour.

\section{Identification of the foraging gene in D. melanogaster} The term foraging describes both food-searching (appetitive) and food-intake (consummatory) processes, encompassing diverse activities that include intensive searching in dogs, ranging in lions, 'theft' in scavengers and ambush strategies of spiders. Foraging behaviours can vary between genotypes within a species, over the life cycle of a single individual, and across environments. The first gene to be cloned that influences natural variation in foodrelated behaviour affects the foraging style of $D$. melanogaster larvae. Natural strains of these larvae exhibit one of two foraging patterns: 'rover' larvae travel further than 'sitter' larvae in a single patch of food and move more between food patches $[18,22]$. In adulthood, the strains retain their separate foraging strategies [23], and rovers also acquire an enhanced sensitivity to sucrose [24]. Notably, rover and sitter locomotion activities do not differ significantly in the absence of food. Genetic and molecular methods showed that the rover-sitter behavioural dimorphism is primarily due to variation at a single gene called foraging (for) that encodes a cGMP-dependent protein kinase (PKG). The finding that PKG levels are higher in rovers than in sitters, combined with a switch to rover-like movement patterns in transgenic sitters (see Glossary) overexpressing PKG, indicates that the difference in sitter appetitive behaviour is due to reduced PKG levels [25].

Although these data convincingly demonstrate a link between PKG levels and natural variation in larval foraging patterns, a neurogenetic understanding of the behaviour requires delineation of the circuitry components responsible for food-related movement. In addition, developmental, neuroanatomical and/or neurophysiological distinctions between rovers and sitters that account for behavioural differences should be investigated. As with CPGs, one challenge is to distinguish those aspects of the wiring that control general locomotion from those that mediate appetitive movements in response to food. The fact that rovers and sitters show similar movement patterns in the absence of food indicates that for must influence a food-specific pathway. However, the broad expression pattern of for and the requirement of PKG for diverse neurobiological processes [26] have made it difficult to identify relevant neurons.

Recent results have shown that the neural substrates important for male fly courtship behaviour are specified by a male-specific splice variant of the fruitless (fru) gene. fru is expressed in all peripheral sensory modalities important for courtship, and in at least one case (olfaction) is required to regulate the perception and response to courtship-relevant environmental cues. An intriguing aspect of fru function is that the circuitry it regulates, which is itself specific for courtship, appears to feed into general elements of the nervous system to direct appropriate mating behaviours $[27,28]$. Whether for acts in an analogous manner to affect circuitry that regulates food-related behaviour in D. melanogaster larvae remains to be seen. One possibility is that for, which expresses at least three transcript types, marks food-specific neural pathways with a single protein isoform. Similar to fru, for function could be associated with multiple circuits (e.g. those of taste, olfaction and/or gut chemosensors) that, when integrated, harness general locomotory machinery to effect the coordinated movement patterns that characterize the appetitive behaviour of a particular strain. 
Given the many physiological factors influencing foodrelated behaviour, PKG might also act outside the nervous system, for example in the gut, fat bodies or ring gland, to influence larval movements and possibly other behaviours such as food intake. In any case, because PKG is not required to induce food-related behavioural changes (rovers, sitters and for mutants all alter their behaviours, albeit in different ways, when moved from non-nutritive media into the presence of food), other molecules no doubt act together with for within food-specific pathways to modify behaviour. Studies have begun to characterize how PKG affects cellular signalling and neuronal function in D. melanogaster $[29,30]$, and future work on strains with mutations converting rovers into sitters [31] and sitters into rovers [32] should identify other molecules necessary for foraging.

\section{Conservation of PKG function across multiple foraging behaviours}

In developmental biology, an emerging theme is that principal regulators of morphology and differentiation have been re-used during evolution to generate novel patterns [33-36]. An intriguing question in behavioural research is whether related behaviours across phylogenetic taxa might also depend on similar molecules and cellular mechanisms. One way to address this issue is using a candidate-gene approach ([37] and references therein) to characterize the roles of genes such as for in the behaviours of other species. This has been done for the honeybee Apis melifera, a social insect in which young adults perform 'nursing' functions in the hive while older animals (2-3 weeks) forage for nectar and pollen outside the nest [38]. Ben-Shahar et al. [39] examined whether the transition from nurse to forager arises from differences in expression of the for honeybee cognate gene (Amfor). They found that foragers had enhanced levels of Amfor mRNA and PKG enzyme activity compared with nurses, even when bees were the same age. Furthermore, experimentally heightening PKG activity in young bees by treatment with cGMP induced precocious foraging, establishing a causative link between Amfor activity and appetitive behaviour.

That manipulation of PKG levels in both honeybees and fruitfly larvae is sufficient to spark dramatic changes in appetitive behaviours galvanizes the hypothesis that common regulators guide related behaviours across taxa. Remarkably, manipulation of PKG does not alter the general locomotion of honeybees or D. melanogaster, and treatment of nurse bees with the structurally similar cAMP produces no effect on foraging [39]. That for appears to regulate specifically the behavioural response to food in two divergent insect species implies that a cGMP pathway has been exploited during evolution to bridge the gap between the requirement for food and appropriate behavioural output.

These results show not only that PKG shares a function in the pathways leading to appetitive behaviours in bees and $D$. melanogaster, but also that variation in the levels of PKG in both species appears to account for natural differences in the expression of the behaviours. In $D$. melanogaster, the source of this variation is at the level of gene sequence: distinct alleles within populations are each associated with a different foraging strategy (rover or sitter). By contrast, in honeybees, single animals titre the level of PKG over their life cycles to regulate foraging activity. In this case, it is unknown whether the impetus provided by increased PKG levels specifically promotes foraging activities such as collecting nectar or whether it influences the nurse-forager transition more generally. It will be interesting to determine whether short-term flux of PKG activity is also used by D. melanogaster larvae to modify feeding behaviour. For example, both rovers and sitters reduce path lengths on food following food deprivation [40], raising the possibility that modulation of PKG levels in individual animals permits balancing of behavioural state with environmental or physiological conditions.

The natural variation observed for PKG levels between genotypes or during development does not necessarily occur for all gene products affecting food-related behaviour. Indeed, the great majority of genes do not vary or respond to change, but are necessary as part of the built-in infrastructure enabling the behaviour to proceed. The functions of such genes must be examined by assessing phenotypes of mutants that have altered gene sequences. For instance, in C. elegans, the for counterpart egl-4 has not yet been associated with natural behavioural variation, but mutational analysis has revealed that here too PKG is required to regulate locomotion in foraging animals [19]. Again, egl-4 does not appear to affect locomotion in the absence of food, indicating that the role of PKG as a specific regulator of appetitive behaviour is conserved in a non-insect species. Future studies should decipher whether PKG also affects foraging behaviour in mammals.

Like many gene products that affect behaviour, PKG has pleiotropic functions throughout the nervous system [26,41]. In D. melanogaster, null for mutations are lethal, and hypomorphic alleles impact foraging, habituation and sucrose responsiveness [24]. In C. elegans, by contrast, egl-4 mutations alter foraging and olfactory adaptation, in addition to body size and lifespan $[19,42,43]$. It is unknown how PKG influences these various processes at the cellular and molecular levels, or how the molecule has become associated with seemingly distinct pathways across evolution. Equally unknown is to what extent different components of PKG pleiotropy, such as foraging and learning, are linked into common modules. Linkage could manifest as common use of the same neural substrates within an organism and maintenance of associations across evolution. One way to address these issues is through the use of microarrays, which should enable comparison of downstream consequences of PKG action in different tissues and species.

\section{Identifying new genes required for food-related behaviours}

In future, a candidate-gene approach could be used to investigate the degree of conservation of other gene functions involved in regulation of food-related behaviour. For example, genetic dissection of CPG function in animals such as C. elegans, D. melanogaster and zebrafish 
has uncovered several important regulators of rhythmic activity [44], opening the door to candidate-gene studies of CPG-controlled feeding behaviours in other animals. In invertebrates such as crustaceans and molluscs that, as already discussed, have well-characterized CPG phenotypes, a candidate-gene approach could provide valuable insights into gene function that then help to elucidate the mechanistic basis of CPG function in other organisms, including $D$. melanogaster, $C$. elegans and mammals. In addition, it will be interesting to determine whether genes such as for, which up to now have been investigated in the context of appetitive foraging behaviour, also have a role in regulation of other food-related processes such as consummation, post-ingestion behaviours or energy homeostasis.

Besides genes previously identified in other species, another source of candidate genes is the microarray, which profiles changes in gene expression associated with different genetic backgrounds or environmental conditions. Complementary-DNA (cDNA) microarrays have been employed extensively to characterize RNA expression patterns in organisms with sequenced genomes, such as humans, D. melanogaster and yeast. However, by using expressed sequence tags (see Glossary) to construct cDNA libraries, genomics approaches have been extended to include organisms not traditionally associated with genetic analysis, including earthworms [45], carp [46], mussels [47] and gobies [48].

By detecting variation in gene activity between different conditions, microarrays can highlight specific genes as potential regulators of a process. For example, Zinke et al. [49] used expression profiles from fooddeprived and carbohydrate-fed flies to identify a gene called sugarbabe (sug) that is activated in response to sugar ingestion. Other uses are diagnostic: Whitfield et al. [50] determined that honeybee nurses and foragers possess distinct transcript 'signatures' that highly correlate with behaviour. By comparing expression profiles of individual bees with those from known nurses and foragers, the identity of bees could be predicted with $95 \%$ accuracy.

One caveat of RNA-based microarrays is the assumption that protein levels within tissues are directly related to transcript abundance, which is not always the case. Thus, in addition to transcript profiling, proteomics methods [51] will help in uncovering the identities, structures and interactions of cellular proteins important for mechanisms of feeding behaviour. Like genetics, these genomics approaches enable identification of key genes and molecules that otherwise might not have been associated with food-related activities.

Of course, to confirm that a gene regulates a process of interest, causation must be established using manipulations that either repress gene function through genetic or post-transcriptional interference (see Glossary) or express gene products constitutively or ectopically using microinjection or transgenic techniques. Pharmacology, antisense technology and RNA interference (RNAi) represent important techniques for inhibiting gene function in organisms not amenable to traditional genetic approaches. For example, in Lymnaea, the enzyme nitric oxide synthase (NOS) is expressed in key modulatory neurons of the feeding network, suggesting that it might be important during feeding [52]. To test this, Korneev et al. [53] performed RNAi targeted against NOS and found a lowered feeding response, indicating that NOS is required for feeding.

Development and application of transgenic techniques in a wider range of species will facilitate further dissection of the molecular mechanisms underlying feeding behaviour in invertebrates $[54,55]$. Transgenic methods confer fine control of neural activity by enabling repression or activation of specific nerve cells [56]. The recent production of 'remote control' flies exhibiting defined behaviours depending on the activity and position of photoactive ion channels demonstrates just how exact such technologies have become [57].

\section{The neurogenetics of food-related behaviour: aggregation in $C$. elegans}

Synthesis of a neurogenetic understanding of food-related behaviour has begun in the genetically tractable system $C$. elegans. Here, instead of superimposing gene function onto a pre-characterized neural phenotype, the general approach is to elucidate roles of genes side-by-side with functions of neurons, thereby gradually building up a comprehensive library of the genetic and neural machinery required for behaviour. Once the regulation and functioning of individual neurons has been established, they can be linked together into circuits that provide mechanistic models of food-related behaviour.

Food-related aggregation [58] is observed in species as diverse as penguins [59], lions [60], hares [61] and even bacteria [62]. Among other advantages, formation of groups facilitates prey capture and repels predators [63]. The neurons and genes underlying group foraging behaviour have begun to be investigated in natural isolates of the nematode $C$. elegans. This species is an excellent system for the development of neurogenetic models of behaviour because the interconnections and lineages of all 302 of its neurons are known [64,65]. Furthermore, using transgenic approaches, gene expression can be restricted to specific neurons at a given stage of development to examine the precise spatial and temporal window when gene action is required [56].

Foraging in C. elegans appears in two forms: feeding alone when placed on a lawn of bacterial food (solitary) and feeding in groups of three to several hundred animals (aggregating) [66]. Aggregating strains also tend to show higher rates of locomotion on food, preference for thicker portions of the bacterial lawn, and more rapid habituation to ethanol $[66,67]$. These differences in behaviour are not found in the absence of food. Remarkably, all of these differences can be explained by variation at a single amino acid within NPR-1, a G-protein-coupled receptor of the neuropeptide Y (NPY) family [66]. As a result of the polymorphism, solitary worms contain NPR-1 receptors that are more active than those in aggregating genotypes. The $n p r-1$ gene appears to be required acutely during foraging, because its activation in only the adult stage of aggregating strains is sufficient to induce solitary behaviour [68]. 


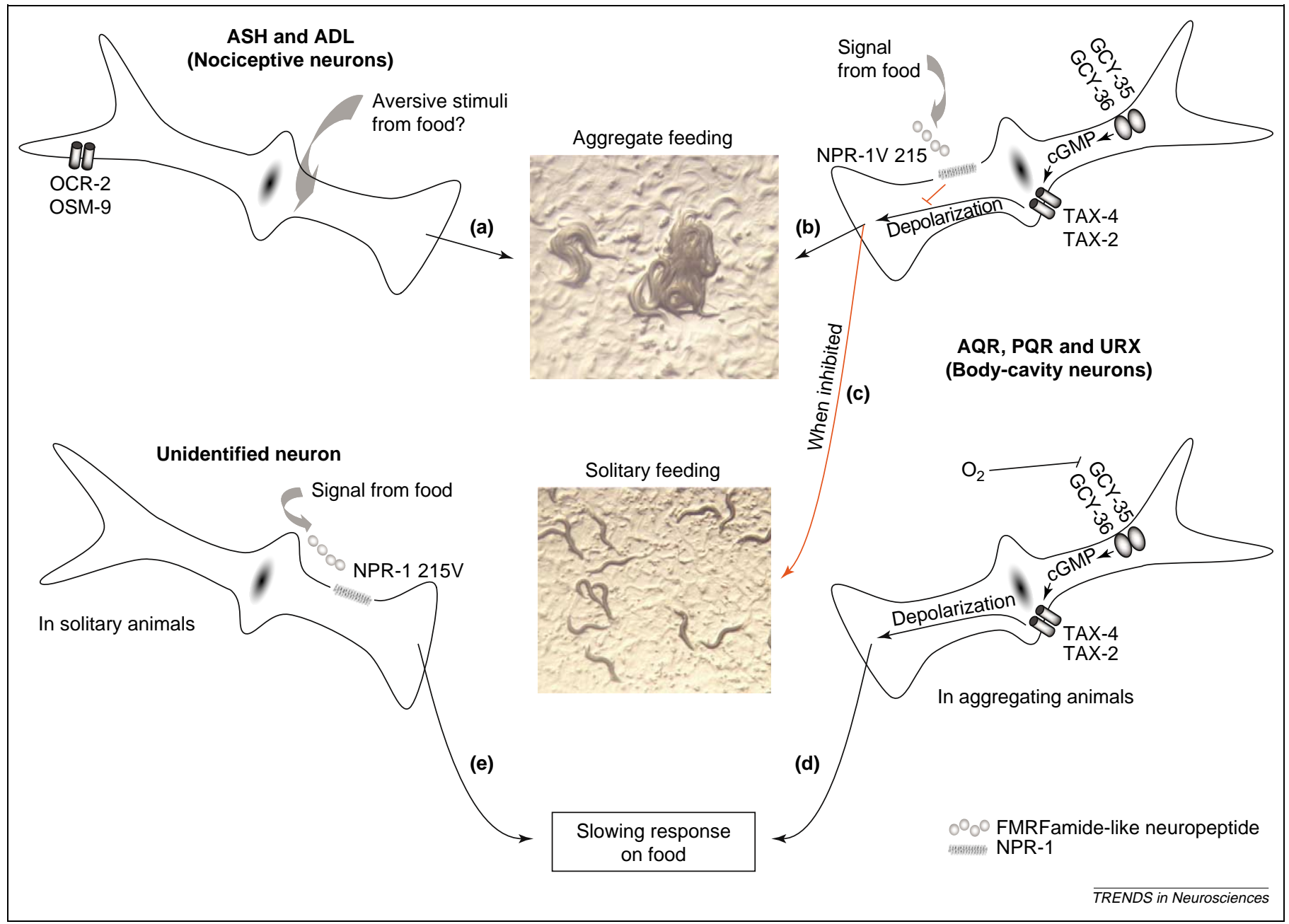

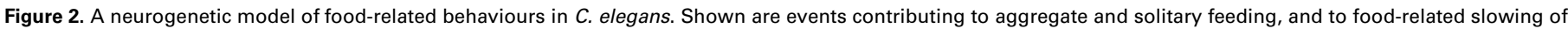

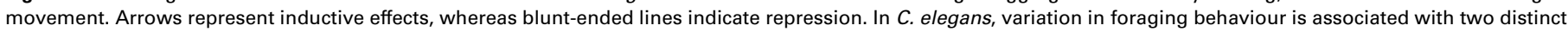

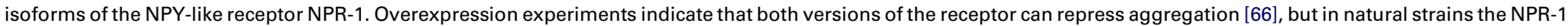

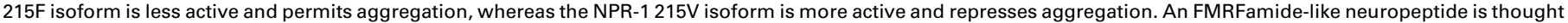

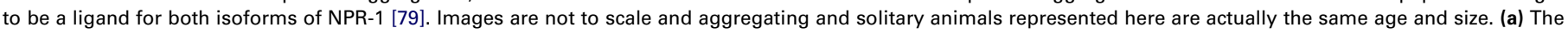
chemosensory neurons ASH and ADL mediate nociception and aggregation during feeding [69]. The ocr-2 and osm-9 genes are required for aggregation; they encode TRPV

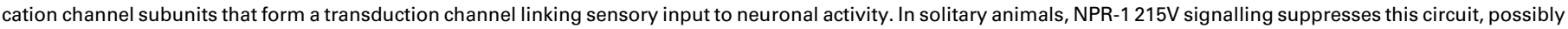

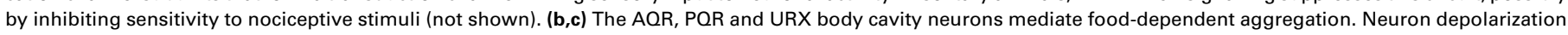
and aggregation depend on a cGMP-dependent signalling pathway that employs the soluble guanylyl cyclases (sGCs) GCY-35 and GCY-36 [75] and a cGMP-gated cation

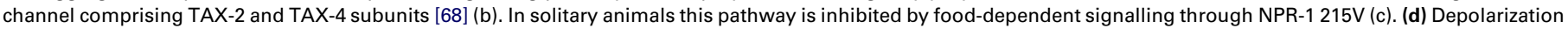

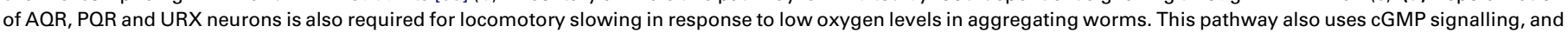

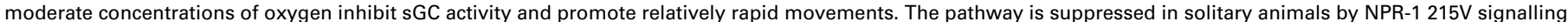

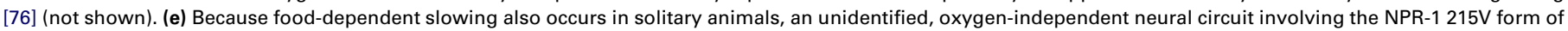
NPR-1 operates to suppress locomotion in response to food. Photographs kindly supplied by Mario de Bono.

One possible reason why $n p r-1$ has such diverse behavioural effects is that, similar to fru in $D$. melanogaster courtship, it acts through multiple neural pathways (Figure 2). One of these circuits involves the chemosensory neurons ASH and ADL, which are required for aggregation and also specialize in mediating aversive responses to noxious stimuli [69]. This connection suggests that group-feeding might be, at least in part, due to perception of aversive cues, conceivably originating from food or the animals themselves. That such a link is not intuitive is a salient instance of the power of genetics to identify regulators without bias and to bring together disparate biological domains.

Induction of solitary behaviour by NPR-1 signalling suggests that this pathway might antagonize responses of $\mathrm{ASH}$ and $\mathrm{ADL}$ to stressful stimuli. Data from other neurobiological contexts support a link between the NPY pathway and nociception, suggesting that such a system might have been adopted through natural selection in C. elegans to regulate foraging behaviour. For example, experiments in rats show that NPY administration in the nucleus accumbens is analgesic [70]. Likewise, mice lacking an NPY receptor experience enhanced aversion to various chemical and mechanical stimuli [71]. Whether other examples of food-related aggregation in nature are associated with nociceptive circuits remains to be determined. Interestingly, NPY has been linked to larval aggregation in D. melanogaster [72], suggesting that, similar to PKG and foraging locomotion, the NPY pathway could have a conserved role in mediating aggregation behaviours.

More generally, it is not surprising that stress interacts with food-related behaviours, because animals have 
limited energy reserves that must be allocated differentially depending on circumstances. For example, D. melanogaster larvae bred in the presence of stressors such as high levels of ammonia or wasp parasitoids show reduced feeding rates and food-related movement relative to unstressed animals [73,74]. Whether energetic tradeoffs exist in aggregating $C$. elegans strains with increased activity of stress-associated neurons remains to be determined. Aggregating and solitary animals should also be observed in their natural environments to determine whether they coexist and, if so, which environmental factors contribute to maintenance of the polymorphism.

In addition to input through chemosensory neurons, $n p r-1$ influences aggregation through a second pathway via cGMP-dependent signalling (Figure 2). Here, depolarization of neurons exposed to body fluid depends on soluble guanylyl cyclases (sGCs; these are responsible for synthesizing cGMP from GTP) and on the tax-2 and tax4 cGMP-gated cation channel subunits [75]. Involvement of cGMP signalling in food-related aggregation highlights remarkable conservation of this pathway as a regulator of diverse foraging activities. In mammals, PKG has also been associated with nociceptive neuron function [26], drawing a further link between cGMP signalling and aggregation behaviour in C. elegans.

Other results suggest that PKG helps build plasticity into foraging behaviour. For example, Cheung and colleagues [76] found that aggregating strains of C. elegans fed in low oxygen levels are conditioned to prefer low oxygen levels when they are later given a choice of different oxygen concentrations. This effect is mediated by sGC activity in body-cavity neurons. Notably, because these neurons are suppressed by NPR1 activity in solitary strains [68,76] (Figure 2), solitary animals fail to respond to depleted oxygen conditions, revealing how a change in gene expression can reconfigure sensory pathways to affect behavioural plasticity [76]. A relationship between PKG and plasticity is also observed in D. melanogaster, where enhanced levels of PKG in rovers versus sitters underlies reduced rates of habituation to a sucrose stimulus [24], in addition to changes in physiological measures of habituation [77]. Finally, in mammals PKG has a central role in mediating plasticity of neurotransmitter release associated with long-term potentiation [26]. Whether plasticity of other food-related behaviours, such as biting in Aplysia, is also regulated by cGMP-PKG-related processes could be investigated using a candidate-gene approach. An intriguing hypothesis is that coupling of cGMP signalling with both foraging and behavioural plasticity builds inherent flexibility into the mechanisms regulating food-related behaviour.

\section{Concluding thoughts}

The great diversity of food-related behaviours within nature ensures that geneticists and neurobiologists, in addition to ecologists, endocrinologists and physiologists, will have new systems to study for some time. Comparative approaches that examine both similarities and distinctions in the regulation of food-related behaviours between species will be necessary to gain an understanding of the specific neural and molecular events that underlie the evolution of food-related behaviours. In addition, it will continue to be useful to focus on a select few species to gain comprehensive knowledge of the way that genes, the environment and their interactions influence nervous system function to produce adaptive feeding behaviours. These organisms must include genetically tractable ones such as mice, Drosophila and C. elegans, but the rich wealth of neural phenotypes available in invertebrates such as molluscs and some crustaceans makes these systems very attractive for development of neurogenetic models of feeding behaviour. Future work will probably highlight the conservation of both molecular and neural principles across various feeding systems. Re-use of the cGMP signalling pathway as a regulator of multiple appetitive behaviours is a striking example of this. Other instances are emerging from nutrient-related homeostasis, where the effects of pathways such as insulin signalling and amino acid deprivation are conserved across the animal kingdom $[78,20]$. As new circuits affecting food-related behaviour continue to be identified, a challenge will be to determine how information from multiple pathways is consolidated and interpreted to trigger appropriate behavioural output. In all, the rich web of interactions contributing to foodrelated behaviour promises to be almost as fascinating as the behaviours themselves.

\section{Acknowledgements}

We thank John Byrne, Mario de Bono and Itay Hurwitz for kindly providing figures and images. We are also grateful to Harold Atwood, Mario de Bono, Ralph Greenspan, Mel Robertson, Gene Robinson and members of the Sokolowski laboratory for valuable comments on the manuscript. In addition we appreciate the insightful suggestions contributed by four anonymous reviewers and the TINS editorial staff. This work was supported by grants to M.B.S. from the Canadian Institute for Health Research, the National Institute of Health and the Canada Research Chair Program.

\section{References}

1 Barsh, G.S. and Schwartz, M.W. (2002) Genetic approaches to studying energy balance: perception and integration. Nat. Rev. Genet. 3, 589-600

2 Bell, C.G. et al. (2005) The genetics of human obesity. Nat. Rev. Genet. 6, 221-234

3 Kamil, A.C. (1987) Foraging Behavior, Plenum Press

4 Alcock, J. (2005) Animal Behavior, 8th edn, Sinauer Associates

5 Dethier, V.G. (1976) The Hungry Fly: A Physiological Study of the Behavior Associated with Feeding, Harvard University Press

6 Stricker, E. and Woods, S.C. (2004) Neurobiology of Food and Fluid Intake, 2nd edn, Kluwer Academic/Plenum

7 Ogden, J. (2003) The Psychology of Eating: from Healthy to Disordered Behavior, Blackwell

8 Nelson, R.J. (2000) Homeostasis and behavior. In An Introduction to Behavioral Endocrinology (2nd edn), pp. 447-496. Sinauer Associates

9 Marder, E. and Bucher, D. (2001) Central pattern generators and the control of rhythmic movements. Curr. Biol. 11, R986-R996

10 Nusbaum, M.P. and Beenhakker, M.P. (2002) A small-systems approach to motor pattern generation. Nature 417, 343-350

11 Elliott, C.J.H. and Susswein, A.J. (2002) Comparative neuroethology of feeding control in molluscs. J. Exp. Biol. 205, 877-896

12 Ayali, A. (2004) The insect frontal ganglion and stomatogastric pattern generator networks. Neurosignals 13, 20-36

13 Miles, C.I. and Booker, R. (1998) The role of the frontal ganglion in the feeding and eclosion behavior of the moth Manduca sexta. J. Exp. Biol. 201, 1785-1798 
14 Hurwitz, I. et al. (2005) Transforming tonic firing into a rhythmic output in the Aplysia feeding system: presynaptic inhibition of a command-like neuron by a CPG element. J. Neurophysiol. 93, 829-842

15 Jones, N.G. et al. (2003) A persistent cellular change in a single modulatory neuron contributes to associative long-term memory. Curr. Biol. 13, 1064-1069

16 Ribeiro, M.J. et al. (2003) Cyclic AMP response element-binding (CREB)-like proteins in a molluscan brain: cellular localization and learning-induced phosphorylation. Eur. J. Neurosci. 18, 1223-1234

17 Brembs, B. et al. (2002) Operant reward learning in Aplysia: neuronal correlates and mechanisms. Science 296, 1706-1709

18 Sokolowski, M.B. (1980) Foraging strategies of Drosophila melanogaster: a chromosomal analysis. Behav. Genet. 10, 291-301

19 Fujiwara, M. et al. (2002) Regulation of body size and behavioral state of $C$. elegans by sensory perception and the EGL-4 cGMP-dependent protein kinase. Neuron 36, 1091-1102

20 Hao, S. et al. (2005) Uncharged tRNA and sensing of amino acid deficiency in mammalian piriform cortex. Science 307, 1776-1778

21 Erickson, J.C. et al. (1996) Attenuation of the obesity syndrome of $o b / o b$ mice by the loss of neuropeptide Y. Science 274, 1704-1707

22 Sokolowski, M.B. et al. (1984) Drosophila larval foraging behaviour: developmental stages. Anim. Behav. 32, 645-651

23 Pereira, H.S. and Sokolowski, M.B. (1993) Mutations in the larval foraging gene affect adult locomotory behavior after feeding in Drosophila melanogaster. Proc. Natl. Acad. Sci. U. S. A. 90, 5044-5046

24 Scheiner, R. et al. (2004) Activity of cGMP-dependent protein kinase (PKG) affects sucrose responsiveness and habituation in Drosophila melanogaster. Learn. Mem. 11, 303-311

25 Osborne, K.A. et al. (1997) Natural behavior variation due to a cGMPdependent protein kinase of Drosophila. Science 277, 834-836

26 Feil, R. et al. (2005) Function of cGMP-dependent protein kinases in the nervous system. Rev. Neurosci. 16, 23-41

27 Manoli, D.S. et al. (2005) Male-specific fruitless specifies the neural substrates of Drosophila courtship behaviour. Nature 436, 395-400

28 Stockinger, P. et al. (2005) Neural circuitry that governs Drosophila male courtship behavior. Cell 121, 795-807

29 Renger, J.J. et al. (1999) Neuronal polymorphism among natural alleles of a cGMP-dependent kinase gene, foraging in Drosophila. J. Neurosci. 19, RC28

30 Riedl, C.A.L. et al. (2005) Drosophila soluble guanylyl cyclase mutants exhibit increased foraging locomotion: behavioral and genomic investigations. Behav. Genet. 35, 231-244

31 Shaver, S.A. et al. (2000) Isolation of larval behavioral mutants in Drosophila melanogaster. J. Neurogenet. 14, 193-205

32 Pereira, H.S. et al. (1995) Chaser (Csr), a new gene affecting larval foraging behaviour in Drosophila melanogaster. Genetics 141, 263-270

33 Reed, R.D. and Serfas, M.S. (2004) Butterfly wing pattern evolution is associated with changes in a Notch/Distal-less temporal pattern formation process. Curr. Biol. 14, 1159-1166

34 Sucena, E. et al. (2003) Regulatory evolution of shavenbaby/ovo underlies multiple cases of morphological parallelism. Nature 424, 935-938

35 Gompel, N. and Carroll, S.B. (2003) Genetic mechanisms and constraints governing the evolution of correlated traits in drosophilid flies. Nature 424, 931-935

36 Carroll, S.B. et al. (2001) From DNA to Diversity: Molecular Genetics and the Evolution of Animal Design, Blackwell

37 Fitzpatrick, M.J. et al. (2005) Candidate genes for behavioural ecology. Trends Ecol. Evol. 20, 96-104

38 Robinson, G.E. et al. (1997) Insect societies and the molecular biology of social behavior. BioEssays 19, 1099-1108

39 Ben-Shahar, Y. et al. (2002) Influence of gene action across different time scales on behavior. Science 296, 741-744

40 Graf, S.A. and Sokolowski, M.B. (1989) Rover/sitter Drosophila melanogaster larval foraging polymorphism as a function of larval development, food-patch quality, and starvation. J. Insect Behav. 2, 301-313

41 Sokolowski, M.B. (2001) Drosophila: genetics meets behaviour. Nat. Rev. Genet. 2, 879-890
42 L'Etoile, N.D. et al. (2002) The cyclic GMP-dependent protein kinase EGL-4 regulates olfactory adaptation in C. elegans. Neuron 36, 10791089

43 Hirose, T. et al. (2003) Cyclic GMP-dependent protein kinase EGL-4 controls body size and lifespan in C. elegans. Development 130, 1089-1099

44 Kullander, K. (2005) Genetics moving to neuronal networks. Trends Neurosci. 28, 239-247

45 Lee, M.S. et al. (2005) Transcriptome analysis in the midgut of the earthworm (Eisenia andrei) using expressed sequence tags. Biochem. Biophys. Res. Commun. 328, 1196-1204

46 Gracey, A.Y. et al. (2004) Coping with cold: an integrative, multitissue analysis of the transcriptome of a poikilothermic vertebrate. Proc. Natl. Acad. Sci. U. S. A. 101, 16970-16975

47 Venier, P. et al. (2003) Towards a catalogue of genes transcribed in multiple tissues of Mytilus galloprovincialis. Gene 314, 29-40

48 Gracey, A.Y. et al. (2001) Hypoxia-induced gene expression profiling in the euryoxic fish Gillichthys mirabilis. Proc. Natl. Acad. Sci. U. S. A. 98, 1993-1998

49 Zinke, I. et al. (2002) Nutrient control of gene expression in Drosophila: microarray analysis of starvation and sugar-dependent response. EMBO J. 21, 6162-6173

50 Whitfield, C.W. et al. (2003) Gene expression profiles in the brain predict behavior in individual honey bees. Science 302, 296-299

51 Choudhary, J. and Grant, S.G.N. (2004) Proteomics in postgenomic neuroscience: the end of the beginning. Nat. Neurosci. 7, 440-445

52 Korneev, S.A. et al. (1998) Molecular characterization of NOS in a mollusc: expression in a giant modulatory neuron. J. Neurobiol. 35, 65-76

53 Korneev, S.A. et al. (2002) Suppression of nitric oxide (NO)-dependent behavior by double-stranded RNA-mediated silencing of a neuronal NO synthase gene. J. Neurosci. 22, RC227

54 Atkinson, P.W. (2002) Genetic engineering in insects of agricultural importance. Insect Biochem. Mol. Biol. 32, 1237-1242

55 Pavlopoulos, A. et al. (2004) Efficient transformation of the beetle Tribolium castaneum using the Minos transposable element: quantitative and qualitative analysis of genomic integration events. Genetics $167,737-746$

56 Wulff, P. and Wisden, W. (2005) Dissecting neural circuitry by combining genetics and pharmacology. Trends Neurosci. 28, 44-50

57 Lima, S.Q. and Miesenböck, G. (2005) Remote control of behavior through genetically targeted photostimulation of neurons. Cell 121, $141-152$

58 Wilson, E.O. (1975) Sociobiology, Belknap Press of Harvard University Press

59 Takahashi, A. et al. (2004) Penguin-mounted cameras glimpse underwater group behaviour. Proc. Biol. Sci. 271(Suppl. 5), S281S282

60 Scheel, D. and Packer, C. (1991) Group hunting behavior of lions - a search for cooperation. Anim. Behav. 41, 697-709

61 Monaghan, P. and Metcalfe, N.B. (1985) Group foraging in wild brown hares: effects of resource distribution and social status. Anim. Behav. 33, 993-999

62 Shimkets, L.J. (1999) Intercellular signaling during fruiting-body development of Myxococcus xanthus. Annu. Rev. Microbiol. 53, $525-549$

63 Giraldeau, L-A. and Caraco, T. (2000) Social Foraging Theory, Princeton University Press

64 White, J.E. et al. (1986) The structure of the nervous system of the nematode Caenorhabditis elegans. Philos. Trans. R. Soc. Lond. B Biol. Sci. 314, 1-340

65 Rankin, C.H. (2002) From gene to identified neuron to behaviour in Caenorhabditis elegans. Nat. Rev. Genet. 3, 622-630

66 de Bono, M. and Bargmann, C.I. (1998) Natural variation in a neuropeptide $\mathrm{Y}$ receptor homolog modifies social behavior and food response in C. elegans. Cell 94, 679-689

67 Davies, A.G. et al. (2004) Natural variation in the $n p r-1$ gene modifies ethanol responses of wild strains of C. elegans. Neuron 42, 731-743

68 Coates, J.C. and de Bono, M. (2002) Antagonistic pathways in neurons exposed to body fluid regulate social feeding in Caenorhabditis elegans. Nature 419, 925-929

69 de Bono, M. et al. (2002) Social feeding in Caenorhabditis elegans is induced by neurons that detect aversive stimuli. Nature 419, 899-903 
$70 \mathrm{Li}$, Y. et al. (2002) Anti-nociceptive effect of neuropeptide Y in the nucleus accumbens of rats: an involvement of opioid receptors in the effect. Brain Res. 940, 69-78

71 Naveilhan, P. et al. (2001) Reduced antinociception and plasma extravasation in mice lacking a neuropeptide $\mathrm{Y}$ receptor. Nature $409,513-517$

$72 \mathrm{Wu}, \mathrm{Q}$.et al. (2003) Developmental control offoraging and social behavior by the Drosophila neuropeptide Y-like system. Neuron 39, 147-161

73 Fellowes, M.D.E. et al. (1999) Association between feeding rate and parasitoid resistance in Drosophila melanogaster. Evolution Int. J. Org. Evolution 53, 1302-1305

74 Mueller, L.D. et al. (2005) Evolution of larval foraging behaviour in Drosophila and its effects on growth and metabolic rates. Phys. Entomol. 30, 262-269
75 Cheung, B.H.H. et al. (2004) Soluble guanylate cyclases act in neurons exposed to the body fluid to promote $C$. elegans aggregation behavior. Curr. Biol. 14, 1105-1111

76 Cheung, B.H.H. et al. (2005) Experience-dependent modulation of C. elegans behavior by ambient oxygen. Curr. Biol. 15, 905-917

77 Engel, J.E. et al. (2000) A cGMP-dependent protein kinase gene, foraging, modifies habituation of the giant fiber escape response in Drosophila. Learn. Mem. 7, 341-352

78 Garofalo, R.S. (2002) Genetic analysis of insulin signaling in Drosophila. Trends Endocrinol. Metab. 13, 156-162

79 Rogers, C. et al. (2003) Inhibition of Caenorhabditis elegans social feeding by FMRFamide-related peptide activation of NPR-1. Nat. Neurosci. 6, 1178-1185

\section{Articles of interest in Current Opinion journals}

The many faces of REST oversee epigenetic programming of neuronal genes

Nurit Ballas and Gail Mandel

Current Opinion in Neurobiology 15, 500-506

Role reversal: the regulation of neuronal gene expression by microRNAs

Matthew E. Klein, Soren Impey and Richard H. Goodman

Current Opinion in Neurobiology 15, 507-513

Glial influences on neural stem cell development: cellular niches for adult neurogenesis

Dengke K. Ma, Guo-li Ming and Hongjun Song

Current Opinion in Neurobiology 15, 514-520

Synaptic protein degradation by the ubiquitin proteasome system Baris Bingol and Erin M. Schuman

Current Opinion in Neurobiology 15, 536-541

Signaling between glia and neurons: focus on synaptic plasticity

Nicola J. Allen and Ben A. Barres

Current Opinion in Neurobiology 15, 542-548

Combining microscience and neurobiology

Douglas B. Weibel, Piotr Garstecki and George M. Whitesides

Current Opinion in Neurobiology 15, 560-567

Semiconductor nanocrystals for biological imaging

Aihua Fu, Weiwei Gu, Carolyn Larabell and A. Paul Alivisatos

Current Opinion in Neurobiology 15, 568-575

Geometric diffusions for the analysis of data from sensor networks

Ronald R. Coifman et al.

Current Opinion in Neurobiology 15, 576-584

Spike train metrics

Jonathan D. Victor

Current Opinion in Neurobiology 15, 585-592

Interrogating rodents regarding their object and spatial memory

Robert E. Clark and Stephen J. Martin

Current Opinion in Neurobiology 15, 593-598

Mapping behaviorally relevant neural circuits with immediate-early gene expression

John F. Guzowski et al.

Current Opinion in Neurobiology 15, 599-606

Protein synthesis-dependent long-term functional plasticity: methods and techniques

Sreedharan Sajikumar, Sheeja Navakkode and Julietta Uta Frey

Current Opinion in Neurobiology 15, 607-613

Integrative neuroscience: linking levels of analyses

Sten Grillner, Alexander Kozlov and Jeanette Hellgren Kotaleski

Current Opinion in Neurobiology 15, 614-62 